When one realizes that this circumstance favours the use of the visible spectrum one can go one step further and consider using the nearby ultra-violet, for which we can also use the eye as a direct indicator with the aid of a fluorescing disc, and for which we have sensitive, non-inert detectors. Today we are in a position to send out a large impulse power of ultra-violet rays in very compact beams and to receive them with such detectors. We could therefore also use them to make a radar instrument. I think such an experiment, using modern materials, might be a worthwhile experiment in order to explore the possibilities.

I should also like briefly to mention the use of reflectors, preferably triple mirrors, which have been the subject of a number of papers and comments. Radar is obviously developing in the direction of microwaves (e.g., $8.6 \mathrm{~mm}$.), which are undoubtedly far superior to infra-red in all respects, especially since in the case of larger objects, such as ships, they give a good image of the shape and position. An obvious step, therefore, seems to be to use suitably mounted reflectors, e.g. on the stern and bows of a ship, to show the aspect of a vessel appearing in the radar area, i.e. her manœuvres. This could, for instance, be done by inclining the reflectors towards each other and observing the relationship in their reflection intensity; this would make it possible to recognize a change of course. Furthermore, such reflectors could be used as transmitters by modulating their reflection. For slow identification the reflector openings could be changed mechanically, perhaps by shutters. But quick modulation with telephone frequencies is also possible with a method which I saw in 1935 in the laboratory of the Radio Corporation of America. A gas discharge valve with a to-and-fro winding, whose current strength is modulated practically without inertia, is placed in the exit plane of the reflector. This can be done by fairly simple means. The ionization in the gas causes a dispersion of the emitted reflected beam which is dependent on the momentary discharge intensity.

Finally, we must consider the question of using the selective gas radiation from the exhaust gases of jet aircraft. It is known that these are partially ionized at the exit opening as a result of the high combustion temperature. This part is indicated by the $3-\mathrm{cm}$. radar wave and so forms a useful additional reflection cross section in addition to that already represented by the metal aircraft itself. If, therefore, one uses radar waves of, e.g., $3 \mathrm{~cm}$. or $8 \mathrm{~mm}$. to irradiate the object, its reverse scatter section appears considerably enlarged compared with the section of its own radiation, which alone would be able to affect an infra-red indicator. The use of infra-red radiation alone for such objects is in my opinion less hopeful than detection by normal radar instruments, especially in thick fog, which would make passive location largely unsafe. It would also be impossible to measure distances, which is done using normal radar with reflected micro and $\mathrm{cm}$. waves.

\title{
Radar and Visibility Distance
}

\section{from Dr. H. Koschmieder}

I suggest that the term we should use instead of Visibility is Range of Visibility, which defines the maximum distance at which a black object can be identified on the horizon. This distance can nowadays be measured. But to my mind even 
that is not good enough. We should be able to record the range of visibility, so as to show whether in fact a ship has been in fog or not. (And that in fact, was, one recalls, a decisive point in the case of the Andrea Doria collision.)

\section{from Prof. G. Simeon \\ (Istituto Universitario Navale, Naples)}

THE range of radar normally used on ships of the Merchant Navy is more than that of small ships, such as fishing boats, \&c. The Regulations specify the range at which navigation lights should be visible for ships of either more or less than 40 tons. The regulations in fact give a definite indication as to the minimum amount of information which would appear to be required for normal navigation. In putting forward proposals which had already been made by the French during the meeting of the French Navigation Institute in Paris in October of last year, and particularly by Messrs. Hugon and Le Moine-Karmor, we would therefore like to propose that there should be placed on these small ships, for use as soon as visibility is lower than what might be considered normal, corner reflectors, which would make them visible on the radar screen at distances not less than the specified range of the lights. In order to reduce as much as possible the effects of interference, two groups might be installed at different heights. Ships which had radar with the minimum characteristics requisite for maritime navigation would then be authorized to move even in fog at a proper speed. These proposals have been stated and illustrated in a memorandum, in Italian, which was presented to the radar conference at Genoa in May 1957.

\section{Radar and the Collision Regulations \\ from Captains G. Jakobsson and A. Lagerwall}

(Swedish Lloyd and the Transatlantic Shipping Company)

WhateVER theoretical and electronic solutions might be recommended and adopted for use on board radar-equipped vessels, we must see to it that in all countries training facilities are established for officers, and that these facilities are also available for senior officers in service who have no other means of learning radar navigation. There is nobody to guide them as to what actions they should take when encountering other vessels in fog. They have to make the decisions themselves, and very often have to make them quickly. The training facilities should be such that they simulate as far as possible actual conditions. Blindfold radar navigation should also be carried out in clear weather and with some responsible person not blindfolded on the bridge to tell the officer at the radar when he is putting the vessel into a dangerous situation. Surprisingly little radar navigation training in this non-hazardous way is carried out on ships at sea in clear weather.

The general view in Sweden is that there is really very little reason to alter or to write new Rules of the Road for radar-equipped vessels. Certain amendments and recommendations, should, however, be made to clarify the existing rules so 